\title{
Role of microenvironmental acidity and tumor exosomes in cancer immunomodulation
}

\author{
Zaira Boussadia ${ }^{1}$, Cristiana Zanetti $^{2}$, Isabella Parolini ${ }^{2}$ \\ ${ }^{1}$ National Center for Drug Research and Evaluation, ${ }^{2}$ Deparment of Oncology and Molecular Medicine, Istituto Superiore di Sanità, Roma, Italy \\ Contributions: (I) Conception and design: Z Boussadia, I Parolini; (II) Administrative support: All authors; (III) Provision of study materials or \\ patients: All authors; (IV) Collection and assembly of data: All authors; (V) Data analysis and interpretation: All authors; (VI) Manuscript writing: All \\ authors; (VII) Final approval of manuscript: All authors. \\ Correspondence to: Zaira Boussadia. National Center for Drug Research and Evaluation, Istituto Superiore di Sanità, Viale Regina Elena 299, Roma \\ 00161, Italy. Email: Zaira.boussadia@iss.it; Isabella Parolini. Deparment of Oncology and Molecular Medicine, Istituto Superiore di Sanità, Viale \\ Regina Elena 299, Roma 00161, Italy. Email: Isabella.parolini@iss.it.
}

\begin{abstract}
Tumor microenvironment (TME) is a complex milieu in which tumor grows, develops and progresses through a complex bi-directional cross-talk with immune-, stromal cells, and the extracellular matrix (ECM). In this context, tumor-derived exosomes (TE) drive the fate of tumor cells through a stimulatory or inhibitory role on immune system. In fact, TE can induce the apoptosis of cells of the immune surveillance, and enhance the proliferation and survival of stromal cells that sustain tumor development. However, depending on the molecular cargo, TE are also able to stimulate anti-tumor immune response. TME is mainly characterized by the acidic $\mathrm{pH}$ that contributes to tumor development, through multiple mechanisms. Among these, the impairment of tumor immune surveillance does occur within acidic TME, and is directly mediated by acidic $\mathrm{pH}$ or by molecular cargo carried by TE. Little is known about the role of TE in immunomodulation in acidic conditions. The present review summarizes the studies describing the role of microenvironmental acidity and TE in immune system modulation.
\end{abstract}

Keywords: Microenvironmental acidity; exosomes; immunomodulation; cancer

Submitted Jan 01, 2020. Accepted for publication Mar 13, 2020.

doi: $10.21037 /$ tcr.2020.03.69

View this article at: http://dx.doi.org/10.21037/tcr.2020.03.69

\section{Introduction}

Tumor mass is characterized by poor blood perfusion, that reduces the supply of oxygen and nutrients, leading to hypoxia, inflammation, enhanced fatty acid metabolism, nucleotide synthesis and glutaminolysis. To support the high proliferation rate, tumor cells are forced to reprogram their metabolism and to increase the rate of glucose uptake, through the up-regulation and the enhanced activity of glucose transporters (1). This process leads to an elevated production of lactate and intracellular accumulation of protons, through aerobic glycolysis, the so-called "Warburg Effect" (2). Additional protons are generated and released by oxygenated cancer cells due the high levels of carbonic dioxide produced during mitochondrial respiration. To avoid cytosolic accumulation of these acidic metabolites and prolonged intracellular acidosis, tumor cells redirect the trans-membrane ion fluxes through vacuolar ATPase (V-ATPase), $\mathrm{Na}+\mathrm{H}+$ exchanger (NHE), monocarboxylate transporters (MCTs), and carbonic anhydrase (3), that actively export protons into extracellular microenvironment, thus lowering the extracellular $\mathrm{pH}$ up to 6.0-6.8. Such hostile milieu selects tumor cells among other non tumor cells that can not adapt.

A large body of evidence indicates that acidosis is a hallmark of almost all tumors (4) as well as a crucial determinant of tumor progression. In fact, it has been reported that, in the early stages of tumor progression, the extracellular acidity influences gene expression by up modulation of several hundred genes encoding receptors, 
signal proteins, transcription factors, cytokines (5), involved in invasion, tissue remodeling, cell cycle control and proliferation $(6,7)$, thus leading to a more malignant cell phenotype.

In this context, the immune cell subsets that are differentially sensitive to low $\mathrm{pH}$, adopt different strategies in order to survive to acidic conditions, but the immune response is impaired by metabolic dysfunctions in cancer cells (8). In fact, under local acidity cancer cells erase the activity of antitumor immune effectors [T cells, natural killer (NK) cells and dendritic cells (DCs)], and at the same time favor the conversion of regulatory $\mathrm{T}\left(\mathrm{T}_{\mathrm{reg}}\right)$ cells and myeloid cells into immunosuppressive and pro-tumor cells. Protons and lactate, create a hostile milieu for $\mathrm{T}$ cells, while myeloid and $\mathrm{T}_{\text {reg }}$ cells can survive thanks to specific metabolic reprogramming and expression of $\mathrm{pH}$ regulators (8).

Tumor cells build a favorable milieu in the microenvironment through a dynamic interplay with stromal and immune cells based on a continuous and abundant release of exosomes. In fact, these nanosized (30$150 \mathrm{~nm}$ ) vesicles endowed with proteins, lipids and nucleic acids (DNA, mRNA, circular RNA, miRNAs and long noncoding RNAs), can be shuttled between neighboring and distant cells, altering the physiological functions of recipient cells.

Tumor exosomes (TE) are involved in a plethora of mechanisms that sustain tumor, such as local invasion, angiogenesis (9-12), preparation of the pre-metastatic niche (13-16), metastasis (17-20) and immune modulation. Depending on their molecular content, TE can either inhibit the host immune system, or enhance anti-tumor immunity. In fact, TE induce $\mathrm{T}$ cell apoptosis, helping tumor cell immune escape (21), and stimulate immune cells against tumor through their endogenous tumor-associated antigens (TAA). These findings paved the way toward the development of anti-tumor vaccines in clinical trials, based on the cross-presentation of tumor $\mathrm{Ag}$ to antigen presenting cells (APCs) $(22,23)$.

Several studies reported the influence of local acidity on the release of exosomes with modified protein content in some tumor models (24-26), likely responsible of disease advancement. However, a direct role of TE released under acidic condition in the modulation of the immune system was never described so far.

Despite growing literature on exosome field of study, however some clarifications are needed. Tumor cells release together with TE a plethora of extracellular vesicles (EVs) of plasma membrane origin (ectosomes) able to elicit biological functions diverse from $\mathrm{TE}$ in target cells. To date, the lack of an elective technical procedure to separate these two populations is a main limitation in the study of TE. Moreover, the complexity of exosomal population has been recently demonstrated, being composed of subpopulations with different size, molecular composition, and biological role (27). All the specific issues in TE/EVs field have been treated in a consensus paper that provides guide lines for the characterization and analysis of EVs subpopulations (28).

In this review, we will provide an overview on the mechanisms of TE on immune modulation, the effects that local acidity may exert on the different players of cancerimmune cross-talk, and the possible role of TE released at low $\mathrm{pH}$ on immune modulation.

\section{Tumor-derived exosomes modulation of immune cells}

It is firmly established that the immune system can be reprogrammed by tumor cells to be ineffective or even acquire tumor-promoting phenotypes. In this scenario, TE play a pivotal role in modulating the immune system, thus driving the fate of tumor development (Table 1). TE can play dual roles-immunostimulatory or immunoinhibitory depending on their molecular content (Figure 1), thus stimulating immune cells against tumor, or inducing $\mathrm{T}$ cell apoptosis, and favoring tumor cell immune escape (57). In the following paragraphs, we will provide an overview of the studies regarding the role of TE in immunomodulation.

\section{Stimulatory role in adaptive immune system}

TE are able to activate immune cells and drive anti-tumor immunity by using the TAA present in their cargo, such as MHC molecules and chaperones (HSP70 and HSP90) $(29,58)$ (Table 1). After internalization in DCs, the cargo of $\mathrm{TE}$ is processed by the antigen processing machinery (APM) and presented to T cells for cellular anti-tumor responses (29).

Notably, ligands carried by TE can be recognized by the cognate receptors on lymphocytes, including the costimulatory molecules CD40, CD80 and CD86, and the adhesion molecule CD54, (59) or, alternatively, TE antigens can bind to cellular MHC receptors. The main mechanism used by exosomes to interact with immune cells is through MHC-I complex, which enables the tumor antigen crosspresentation mediated by DCs, and the activation of T cells response, thus inducing tumor cells death $(30,60)$. 
Table 1 List of representative TE molecules involved in stimulatory and inhibitory functions in immune cells

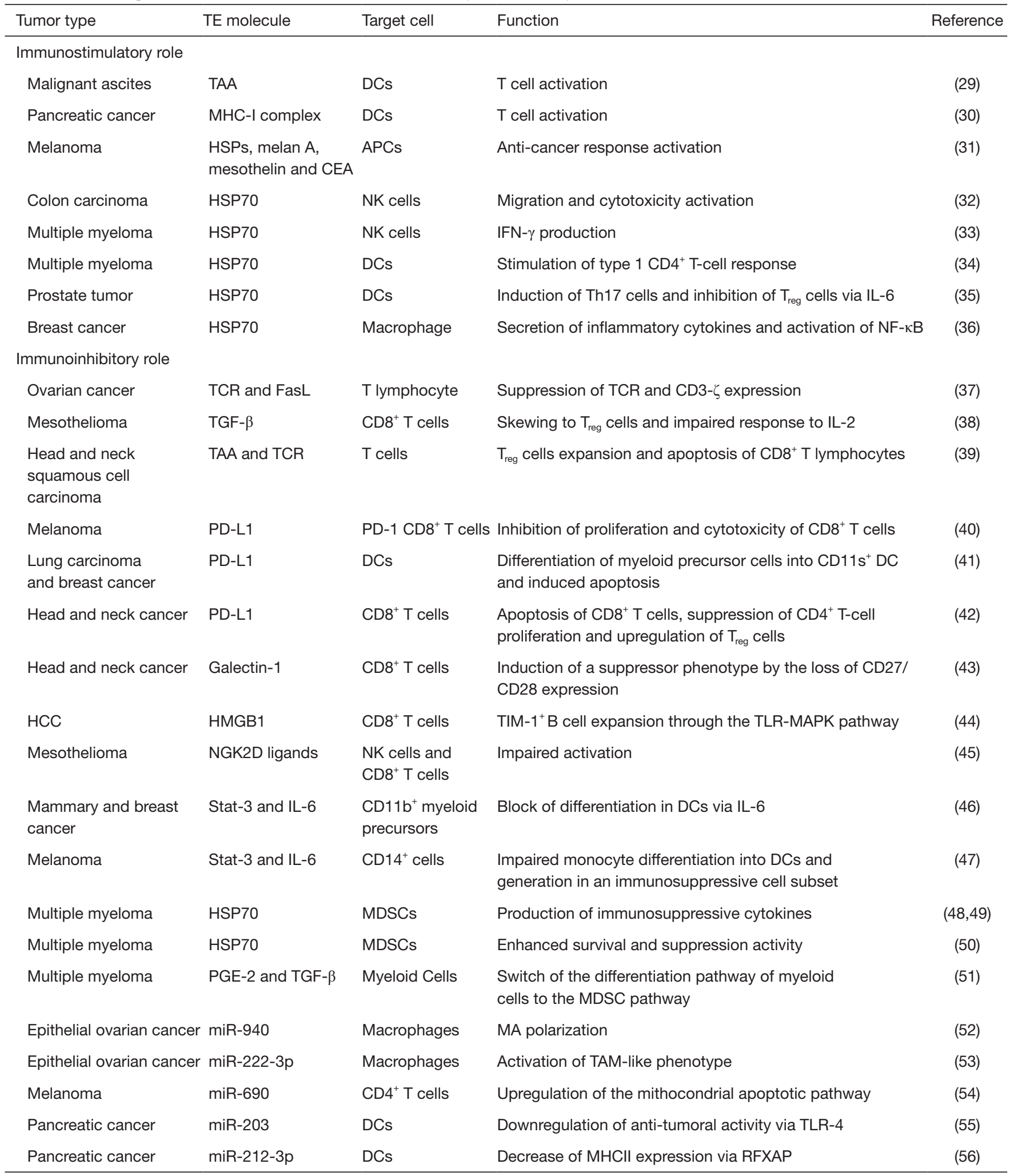

TE, tumor exosome; TAA, tumor-associated antigens; DCs, dendritic cells; APC, antigen presenting cell; CEA, carcinoembryonic antigen; NK, natural killer; $\mathrm{T}_{\text {reg }}$, regulatory T; TCR, T-cell receptor; MDSCs, myeloid-derived suppressor cells. 

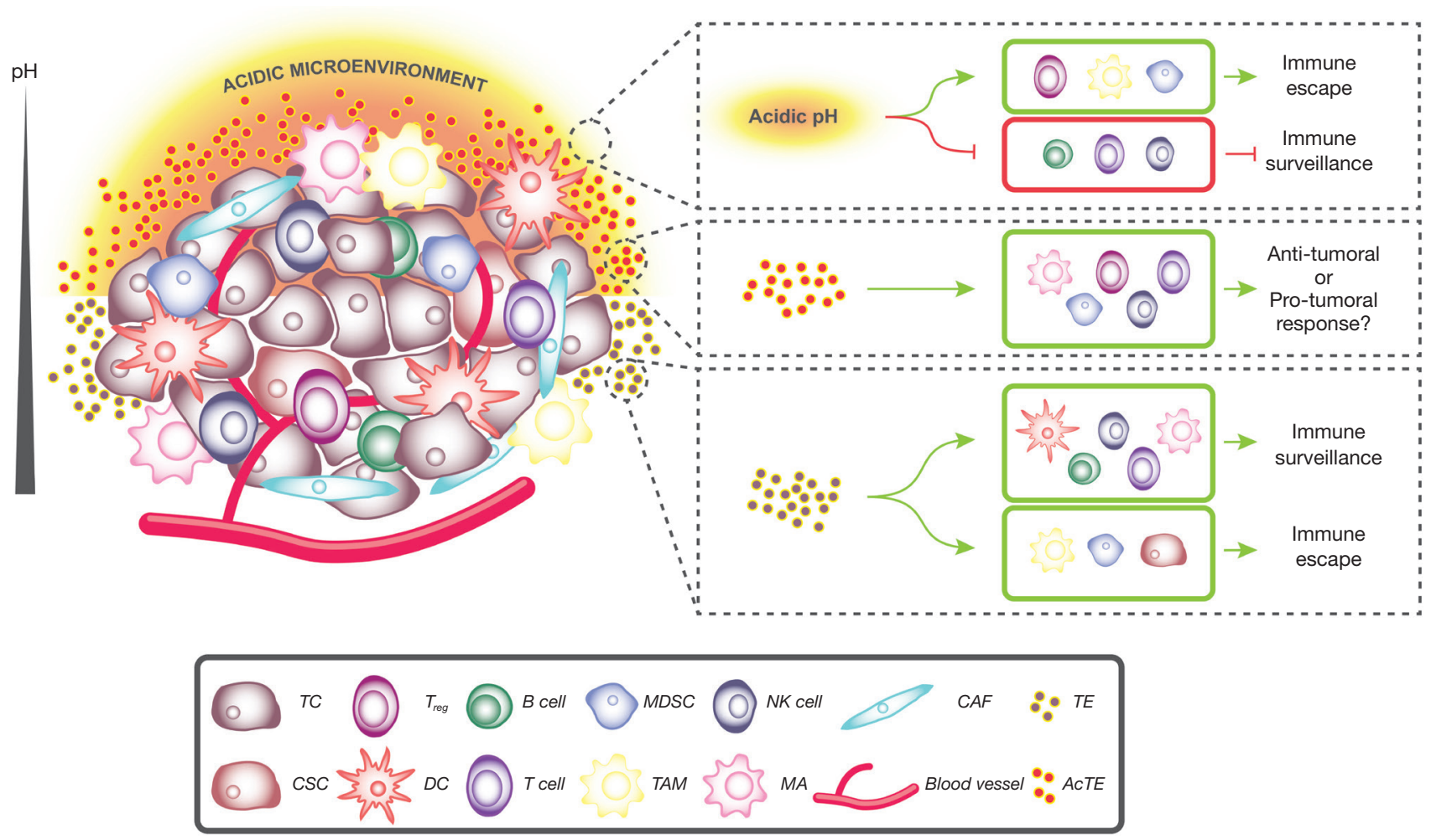

Figure 1 Schematic representation of immune modulation within TME. Cancer cells interact with immune cells through exosomes that can mediate either anti- or pro-tumor responses. Moreover, low $\mathrm{pH}$ per se is able to modulate different immune cells that promote the cancer immune escape, helping cancer progression and growth. Acidic tumor exosomes can play a role in immune modulation, activating multiple cell subsets. B, B cells; CAF, cancer-associated fibroblasts; CSC, cancer stem cells; DCs, dendritic cells; MA, macrophages; MDSC, myeloidderived suppressor cells; NK, natural killer cells; $\mathrm{T}_{\text {reg }}$, regulatory $\mathrm{T}$ cells; TAM, tumor-associated macrophages; TC, tumor cells; TE, tumor exosomes; AcTE, tumor exosomes released in acidic TME; TME, tumor microenvironment.

\section{Stimulatory role in innate immune cells}

TE proteomic analysis revealed that several cancer-specific antigens such as melan $\mathrm{A}$, mesothelin and carcinoembryonic antigen (CEA), are responsible of the exosome-mediated immune surveillance, through the activation of APCs that support the anti-cancer immune response $(31,60)$.

Besides indirect presentation, exosomes can also directly activate macrophages, neutrophils, NK cells and APCs. Notably, TE carrying HSP70 are able to induce NK cells migration and cytotoxicity activity (32) and the production of interferon- $\gamma(\mathrm{IFN}-\gamma)(33)$. Moreover, the binding of HSP70 to TLR2 stimulates DCs $(34,35)$ and macrophages $(36,61)$, that in turn activate $\mathrm{T}$ cell response and NK cellmediated immunity.

In addition, $\mathrm{TE}$ create a local inflammatory environment that enhances an immune response, thus representing a powerful adjuvant in anti-cancer therapies (62).
Unfortunately, the capability of TE to stimulate the immune system is not always sufficient to prevent tumor progression, perhaps due to the dual opposite role exerted by these vesicles in the tumor microenvironment (TME).

\section{Inhibitory role in adaptive immune system}

TE are endowed with molecules (Table 1), which are able to interact with immune cells and suppress their antitumor activity. This process leads to a pro-tumorigenic microenvironment, resulting in an enhancement of tumor growth and shorter survival (44).

It has been reported that TE can induce the apoptosis of $\mathrm{T}$ lymphocytes and induce the loss of the expression of TCR-associated signal transducing $\zeta$-chain, causing an impaired $\mathrm{T}$ cell-mediated immune responsiveness in cancer patients (37). TE can also inhibit the IL-2 proliferative response in $\mathrm{CD}^{+} \mathrm{T}$ cells and favor $\mathrm{T}_{\text {reg }}$ cell responses $(38,39)$. 
Another systemic immune-inhibitory mechanisms used by $\mathrm{TE}$ is represented by activation of apoptosis in $\mathrm{CD} 8^{+}$ and $\mathrm{CD}^{+} \mathrm{T}$ cells, mediated by the exosomal FasL or PDL1 (40,63-66). In addition, Czystowska and colleagues demonstrated that $\mathrm{T}$ cell apoptosis, mediated by $\mathrm{TE}$ containing FasL, is significantly inhibited by $\mathrm{T}$ cells pretreatment with IRX-2, a cytokine-based biological agent, thus suggesting its possible usage in cancer biotherapies (67).

In head and neck cancer, $\mathrm{CD} 8^{+} \mathrm{T}$ lymphocytes were sensitive to apoptosis via PD-L1 pathway, likely responsible of a reduced immune competence in cancer patients $(42,68)$. Moreover, in lung carcinoma and breast cancer it has been shown, that exosomal PD-L1 block the differentiation of $\mathrm{CD} 4^{+} \mathrm{IFN}-\gamma^{+} \mathrm{Th} 1$ cells, inhibit the maturation and migration of DCs and induce their apoptosis (41). Hence, $\mathrm{PD} 1 / \mathrm{PD}-\mathrm{L} 1$ is a key regulator of immune response in $\mathrm{T}$ cells and its inhibition represents a treatment for some tumors (69). Accordingly, Chen and colleagues, indicated a correlation between a higher level of circulating exosomal PD-L1 and a poorer clinical outcomes (40), thus providing a rationale for the use of exosomal PD-L1 in cancer therapy.

TE can impair the immune surveillance mediated by $\mathrm{CD} 8^{+} \mathrm{T}$ cells through several pathways in different tumor models. In head and neck cancer, TE affect CD8 ${ }^{+}$ $\mathrm{T}$ cells by inducing a loss of CD27/CD28, together with decreased levels of the antitumor cytokine IFN- $\gamma(43)$. In hepatocellular carcinoma (HCC), $\mathrm{CD} 8^{+} \mathrm{T}$ cells activity may be down-regulated via the TLR2/4-MAPK pathway, triggered by exosomes containing high mobility group box 1 (HMGB1), in turn leading to immune surveillance failure (44).

Finally, in a melanoma model, exosomal miRNA-690, targeting Rab27a, transferred to $\mathrm{CD}^{+} \mathrm{T}$ cells accelerated mitochondrial apoptosis by down-regulating the expression of anti-apoptotic proteins, such as B-cell lymphoma-2 (BCL-2), induced myeloid leukemia cell differentiation protein (Mcl-1) and B-cell lymphoma-extra large (BCL-xL) (54).

\section{Inbibitory role in innate immune system}

Recent studies indicated that TE can also affect NK cells. Ligands, such as MIC-A/B, ULBP-1/2/3, carried by TE are able to inhibit the expression of receptors NKG2D, NKP30 and NKP46, that activate NK, thus impairing NK cytotoxic activity $(45,70)$. NK cell function can also be regulated by exosomes through TGF- $\beta$ signaling (71).

TE interfere also with the activity and numbers of
DCs. The decrease of DCs activity is mediated by the phosphorylation of Stat-3 and the expression of IL-6, that inhibits also the differentiation of $\mathrm{CD} 14^{+}$monocytes into DCs $(46,47)$. In this context, CD $14^{+}$cells skew their differentiation toward immunosuppressive CD14 ${ }^{+}$HLADR-/low cells, which release TGF- $\beta$ to inhibit T cells (47). Also exosomal miRNAs are able to modulate multiple pathway of DC, such as TLR4 $(55,72)$ and the regulatory factor X-associated protein (RFXAP) (56), in order to down-regulate immune activity, thereby promoting proliferation and migration of cancer cells.

Other key players in inflammation during disease progression are macrophages (MA). TE through miR940 present in their cargo, can modify the polarization of macrophages (M2), promoting cancer cell proliferation and migration (52). It has also been described, in epithelial ovarian cancer, that exosomal miR-222-3p is able to target MA, inducing a switch to a tumor-associated macrophage (TAM)-like phenotype (53).

\section{Inbibitory role in myeloid-derived suppressor cells}

Myeloid-derived suppressor cells (MDSCs), a heterogeneous population of immune cells (73), are key players in immune escape of cancer. The increased numbers of MDSCs in neoplastic lesions are correlated with the neoplastic progression and decreased patient survival, through the inhibition of $\mathrm{NK}, \mathrm{CD}^{+}$and $\mathrm{CD} 8^{+}$ lymphocytes $(42,74,75)$. Within TME, MDSCs produce numerous immunosuppressive inhibitory factors, including nitric oxide (NO) and reactive oxygen species (ROS), which cause T-cell apoptosis, T cell receptor (TCR) nitration (76), and depletion of arginine and cysteine, preventing T-cell activities (77).

TE are able to promote the survival and activity of MDSCs (50,51), which in turn secrete exosomes that can polarize monocytes to $\mathrm{M} 2$ phenotype, inducing the formation of a tumor-promoting microenvironment (78). TE modulate MDSCs through PGE-2, TGF-B, IL-2 and IL-10, and miRNAs such as miR-21, miR-10a, miR-155, miR-126-3p, miR-27b, miR-320, and miR-342-3p and miR-29a [for a detailed review see (79)].

Other studies reported that MDSCs after the activation by exosomal HSP70 and HSP72 can induce the production of immunosuppressive cytokines, through TLR2 engagement $(48,49)$. Therefore, this immunoinhibitory role of HSPs seems in contraddiction with the immunostimulatory activity reported on NK (80-82). This 
can be related to the interaction of HSPs with immune cell receptors with divergent properties.

In conclusion within TME, TE play a key role in the tumor immune escape, thus allowing tumor proliferation and metastatization in secondary sites. This occurs through multiple mechanisms and interactions with immune cells, leading to apoptosis of T cells, impairment of NK, DCs, MA activities, to reduce their anti-tumor role and down-regulating inflammation. This in turn leads to the recruitment of cells that inhibit the anti-tumor responses such as TAM, $\mathrm{T}_{\text {reg }}$ and MDSCs.

\section{Role of microenvironmental acidosis in immune cells}

\section{Adaptive immune system}

Acidosis within TME plays an important role in tumor progression and metastasis, by promoting angiogenesis and invasion and playing a role in cancer immune escape (83) (Figure 1).

TME acidification may affects the anti-tumor response of immune cells, through the metabolic competition between $\mathrm{T}$ and tumor cells and extracellular accumulation of lactate and/or H+. Pathological concentrations of lactic acid are associated with $\mathrm{T}$ and NK cells inhibition, leading to poor outcome in tumor patients (84). This can be explained by the capacity of lactic acid to inhibits the upregulation of nuclear factor of activated $\mathrm{T}$ cells (NFAT) in $\mathrm{T}$ and NK cells, resulting in diminished IFN- $\gamma$ and cytokines production (83). These cytokines are known to sustain antitumor immunity, and develop long-term immunological memory (8). Therefore, the decreased IFN- $\gamma$ secretion under acidosis can determine a failure of the immune surveillance.

Another study reported that low $\mathrm{pH}$ values (6-6.5), cause anergy in both human and mouse tumor-specific CD $8^{+}$ T lymphocytes, thus decreasing the cytolytic activity, the expression of the $\alpha$-chain of the interleukin- 2 receptor (IL$2 \mathrm{R} \alpha$ ) and T-cell receptor (TCR), as well as a diminished activation of STAT5 and ERK in response to TCR signalling (85).

\section{Innate immune system and MDSCs}

It is well established that acidity, is a major "attractor" of myeloid cells to tumor site, and contributes to alter their anti-tumor response. In fact, a study indicated in prostate cancer, that extracellular tumor acidity $(\mathrm{pH}$ 6.8) contributes to carcinogenesis by altering the state of macrophage infiltration, through the modulation of MHC I (86). In another study, lactic acid was described to induce M2like polarization of TAMs through HIF- $1 \alpha$, arginase I and VEGF (87).

Other studies about the activation of DCs in acidic TME highlight conflicting evidences. It was reported that extracellular acidosis improves the capacity of DCs to Ag-presentation, together with the induction of $\mathrm{CD} 8^{+}$ cytotoxic T lymphocytes (CTLs) responses in vivo (88). These effects are probably mediated by acid-sensing ion channels (ASICs) (89). On the contrary, other studies described that extracellular acidosis induces in DCs a tolerogenic phenotype and an impaired migratory response to CCL-19, a lymph node-derived chemokine $(90,91)$, thereby triggering an immunosuppressive state within TME.

Acidity is also a direct regulator of MDSCs survival and activity in TME. MDSCs are attracted to the tumor site by selective inflammatory chemokines (e.g., CCL-2 and CCL-5) and homing factors. When MDSCs reach the tumor site, they mediate immune tolerance and promote tumor growth by favoring angiogenesis, matrix remodeling and epithelial to mesenchymal transition (EMT). MDSCs within TME can differentiate into TAMs or tumorassociated neutrophil (TANs), thus preserving their protumor inflammatory properties (92).

\section{Exosomes released in acidic condition as potential modulators of immune cells}

In the acidic TME, TE play a key role in tumor development through the continuous exchanges of molecular content between cancer and stromal cells. These intercellular communications can drive metastatic properties either at intratumoral level or at distant sites by means of pre-metastatic niche formation and metastases onset (93). It was reported that acidic pressure increased the release of TE in several tumors (prostate, colorectal, lung, melanoma) $(7,94,95)$ and the efficiency transfer of exosomes in target cells (24). As a consequence, recipient cells enhance proliferation, local invasion and metastasis.

A precise role of $\mathrm{TE}$ released at acidic $\mathrm{pH}$ was recently described in a human melanoma model (26). In this study, by means of an innovative cell labeling strategy it was possible to estimate in acidic condition the enhanced release of exosomes endowed with pro-migratory and pro- 
invasive molecules: proto-oncogenes (HRAS, NRAS), metalloprotease (TIMP3), heath shock protein isoforms (HSP90AB1, HSP90B1, HSPAIL, HSPA5), enzyme (GANAB), and actin-binding proteins (GSN, CFL2). To date, these molecules are involved in signaling pathways triggered in tumor advancement.

Interestingly, it was reported a role for some of these proteins in tumor immune modulation (Figure 1). For instance, in thyroid cancer the activation of HRAS together with the loss of PTEN increased the secretion of SDF-1, I-TAC, CCL9/10, and MCP5. These cytokines induced the chemotaxis of MA, MDSCs, and Foxp $3^{+} \mathrm{T}_{\text {reg }}$ cells at the tumor stroma, and the secretion of factors that support tumor cell growth and angiogenesis, ultimately leading to tumor progression and metastasis (96).

In breast cancer, GSN was associated to late stage of the tumor, and induced both a Th1 $\mathrm{CD}^{+}$and $\mathrm{CD} 8^{+} \mathrm{T}$ cell immune response against the tumor (97). HSPs on the surface of exosomes are recognized by $\mathrm{CD} 91^{+}$tumor cells and fibroblasts, CD91 ${ }^{+} \mathrm{SREC1}^{+} \mathrm{TLR}^{+}$professional APCs and $\mathrm{CD} 94^{+}$cytolytic immune cells, thus driving anti-cancer responses. Therefore, HSPs can activate malignancy events in tumor cells and, in contrast, can trigger antigen crosspresentation and cross-priming by APCs and stimulate the cytolytic immune cells [for a detailed review see (98)]. High expression of HSP90 was reported in several cancer models, (lung, breast, colon, and blood), and correlates with poor prognosis $(99,100)$. Hence, targeting HSP70/90 resulted in reduced induction of immune suppressive cells, e.g., preventing the monocyte differentiation into suppressive immune cells (101).

Altogether, these studies provide evidences that acidic TME induces an abundant release of exosomes, whose specific molecular content has the potential to modulate immune cell functions.

\section{Conclusions}

TME actively regulates the onset and growth of the tumor (102), principally through an impairment of the immune system. One of the features of TME is acidity, which takes place in tumor cells after the metabolic switch to aerobic glycolysis, and drives tumor proliferation and metastatization.

It has been described that TME acidity, and especially lactate, is a central regulator of cancer immunity, able to coordinate both local and systemic immunosuppression, thus preventing activation of anti-tumor responses. This deregulation derives from multiple mechanisms that shorten the lifespan or inhibit the proliferation of several immune cell types, such as $\mathrm{CD}^{+}$and $\mathrm{CD} 8^{+}$CTLs, tumor infiltrating CTLs, DCs and NK cells (8).

The study of the impact of acidic microenvironment on immune cells represents an emerging field of research, and it can provide tools to identify promising candidates for immunomodulation in clinical setting. For instance, several pre-clinical and clinical trials examined the impairment of lactate homeostasis as a possible approach for cancer therapeutics (103). The treatment with lactate inhibitors in synergy with other adjuvant therapies might be applied also to potentiate the efficacy of tumor immunotherapy (104).

The dysfunction of the host's immune system represents one of the major mechanisms by which tumors evade immunosurveillance. This impairment can be mediated by TE that have the ability to interact with various factors and modify the phenotype of recipient cells.

The powerful role of these vesicles in the development of tumors has been clearly addressed at several phases during carcinogenesis, in microenvironment reprogramming, premetastatic niche formation (a step required for tumor cell dissemination in target organs), and apoptosis or inhibition of immune cells of innate and adaptive immunity. Hence, depending on cargo and origin, TE can also stimulate the immune anti-tumor response, paving the way for the use of exosomes on immune therapy. However, further studies are needed to produce exosomes that fit the requirements to mediate strong co-stimulatory signals.

The two apparent divergent functions of TE on immune system can be explained by the existence of subpopulations of TE with stimulatory or inhibitory molecules, which can alter the functional status of different immune system components within the TME.

It is worth notice that, TE immune stimulation is an important feature for his use as anti-cancer vaccines. In fact, modifying APC or mesenchymal stem cells (MSCs) function in vivo or in vitro to facilitate release of immunosuppressive EVs could be used to enhance the cytotoxic response against cancer $(105,106)$.

Little is known about the functions exerted by TE secreted within the acidic TME. In melanoma, exosomes released under acidosis contain specific pro-invasive and pro-migratory molecules, which correlated with disease poor prognosis. This enlightened the increased release of acidic $\mathrm{TE}$ as a mechanism required for tumor advancement.

Several molecules specifically included within acidic exosomal cargo were described to modulate the immune 
cells. However, some of these molecules have been described to mediate an anti-cancer response and others to have pro-tumoral functions. Therefore, further investigation are needed to unveil a possible role of acidic TE in immunomodulation.

\section{Acknowledgments}

We thank Luigi Suglia for his help in the production of the figure.

Funding: Funding for this work was provided by the Italian Ministry of Health (Ricerca Corrente).

\section{Footnote}

Provenance and Peer Review: This article was commissioned by the Guest Editor (Jia Wei) for the series "Tumor immune microenvironment in cancer progression and cancer therapy" published in Translational Cancer Research. The article was sent for external peer review organized by the Guest Editor and the editorial office.

Conflicts of Interest: All authors have completed the ICMJE uniform disclosure form (available at http://dx.doi. org/10.21037/tcr.2020.03.69). The series "Tumor immune microenvironment in cancer progression and cancer therapy" was commissioned by the editorial office without any funding or sponsorship. The authors have no other conflicts of interest to declare.

Ethical Statement: The authors are accountable for all aspects of the work in ensuring that questions related to the accuracy or integrity of any part of the work are appropriately investigated and resolved.

Open Access Statement: This is an Open Access article distributed in accordance with the Creative Commons Attribution-NonCommercial-NoDerivs 4.0 International License (CC BY-NC-ND 4.0), which permits the noncommercial replication and distribution of the article with the strict proviso that no changes or edits are made and the original work is properly cited (including links to both the formal publication through the relevant DOI and the license). See: https://creativecommons.org/licenses/by-nc-nd/4.0/.

\section{References}

1. Webb BA, Chimenti M, Jacobson MP, et al. Dysregulated
$\mathrm{pH}$ : a perfect storm for cancer progression. Nat Rev Cancer 2011;11:671-7.

2. Warburg O. On the origin of cancer cells. Science 1956;123:309-14.

3. Spugnini EP, Sonveaux P, Stock C, et al. Proton channels and exchangers in cancer. Biochim Biophys Acta 2015;1848:2715-26.

4. Fais S, Venturi G, Gatenby B. Microenvironmental acidosis in carcinogenesis and metastases: new strategies in prevention and therapy. Cancer Metastasis Rev 2014;33:1095-108.

5. Fukamachi T, Ikeda S, Wang X, et al. Gene expressions for signal transduction under acidic conditions. Genes (Basel) 2013;4:65-85.

6. Moellering RE, Black KC, Krishnamurty C, et al. Acid treatment of melanoma cells selects for invasive phenotypes. Clin Exp Metastasis 2008;25:411-25.

7. Rofstad EK, Mathiesen B, Kindem K, et al. Acidic extracellular $\mathrm{pH}$ promotes experimental metastasis of human melanoma cells in athymic nude mice. Cancer Res 2006;66:6699-707.

8. Huber V, Camisaschi C, Berzi A, et al. Cancer acidity: An ultimate frontier of tumor immune escape and a novel target of immunomodulation. Semin Cancer Biol 2017;43:74-89.

9. Tang MKS, Yue PYK, Ip PP, et al. Soluble E-cadherin promotes tumor angiogenesis and localizes to exosome surface. Nat Commun 2018;9:2270.

10. Gong M, Yu B, Wang J, et al. Mesenchymal stem cells release exosomes that transfer miRNAs to endothelial cells and promote angiogenesis. Oncotarget 2017;8:45200-12.

11. Lee HY, Chen CK, Ho CM, et al. EIF3C-enhanced exosome secretion promotes angiogenesis and tumorigenesis of human hepatocellular carcinoma. Oncotarget 2018;9:13193-205.

12. Todorova D, Simoncini S, Lacroix R, et al. Extracellular vesicles in angiogenesis. Circulation Research 2017;120:1658-73.

13. Peinado H, Zhang H, Matei IR, et al. Pre-metastatic niches: organ-specific homes for metastases. Nat Rev Cancer 2017;17:302-17.

14. Costa-Silva B, Aiello NM, Ocean AJ, et al. Pancreatic cancer exosomes initiate pre-metastatic niche formation in the liver. Nat Cell Biol 2015;17:816-26.

15. Plebanek MP, Angeloni NL, Vinokour E, et al. Premetastatic cancer exosomes induce immune surveillance by patrolling monocytes at the metastatic niche. Nat Commun 2017;8:1319. 
16. Guo Y, Ji X, Liu J, et al. Effects of exosomes on premetastatic niche formation in tumors. Mol Cancer 2019; 18:39.

17. Ruivo CF, Adem B, Silva M, et al. The biology of cancer exosomes: insights and new perspectives. Cancer Res 2017;77:6480-8.

18. Lobb RJ, Lima LG, Möller A. Exosomes: key mediators of metastasis and pre-metastatic niche formation. Semin Cell Dev Biol 2017;67:3-10.

19. Kahlert C, Kalluri R. Exosomes in tumor microenvironment influence cancer progression and metastasis. J Mol Med 2013;91:431-7.

20. Dos Anjos Pultz B, Andrés Cordero da Luz F, Socorro Faria $\mathrm{S}$, et al. The multifaceted role of extracellular vesicles in metastasis: priming the soil for seeding. Int J Cancer 2017;140:2397-407.

21. Syn N, Wang L, Sethi G, et al. Exosome-mediated metastasis: from epithelial-mesenchymal transition to escape from immunosurveillance. Trends Pharmacol Sci 2016;37:606-17.

22. Escudier B, Dorval T, Chaput N, et al. Vaccination of metastatic melanoma patients with autologous dendritic cell (DC) derived-exosomes: results of thefirst phase I clinical trial. J Transl Med 2005;3:10.

23. Morse MA, Garst J, Osada T, et al. A phase I study of dexosome immunotherapy in patients with advanced nonsmall cell lung cancer. J Transl Med 2005;3:9.

24. Parolini I, Federici C, Raggi C, et al. Microenvironmental $\mathrm{pH}$ is a key factor for exosome traffic in tumor cells. J Biol Chem 2009;284:34211-22.

25. Logozzi M, Spugnini E, Mizzoni D, et al. Extracellular acidity and increased exosome release as key phenotypes of malignant tumors. Cancer Metastasis Rev 2019;38:93-101.

26. Boussadia Z, Lamberti J, Mattei F, et al. Acidic microenvironment plays a key role in human melanoma progression through a sustained exosome mediated transfer of clinically relevant metastatic molecules. J Exp Clin Cancer Res 2018;37:245.

27. Zhang H, Freitas D, Kim HS, et al. Identification of distinct nanoparticles and subsets of extracellular vesicles by asymmetric flow field-flow fractionation. Nat Cell Biol 2018;20:332-43.

28. Théry C, Witwer KW, Aikawa E, et al. Minimal information for studies of extracellular vesicles 2018 (MISEV2018): a position statement of the International Society for Extracellular Vesicles and update of the MISEV2014 guidelines. J Extracell Vesicles 2018;7:1535750.
29. Andre F, Schartz NEC, Movassagh M, et al. Malignant effusions and immunogenic tumour-derived exosomes. Lancet 2002;360:295-305.

30. Zeng F, Morelli AE. Extracellular vesicle-mediated MHC cross-dressing in immune homeostasis, transplantation, infectious diseases, and cancer. Seminars in Immunopathology 2018;40:477-90.

31. Mears R, Craven RA, Hanrahan S, et al. Proteomic analysis of melanoma-derived exosomes by twodimensional polyacrylamide gel electrophoresis and mass spectrometry. Proteomics 2004;4:4019-31.

32. Gastpar R, Gehrmann M, Bausero MA, et al. Heat shock protein 70 surface-positive tumor exosomes stimulate migratory and cytolytic activity of natural killer cells. Cancer Res 2005;65:5238-47.

33. Vulpis E, Cecere F, Molfetta R, et al. Genotoxic stress modulates the release of exosomes from multiple myeloma cells capable of activating NK cell cytokine production: role of HSP70/TLR2/NF-kB axis. Oncoimmunology 2017;6:e1279372.

34. Xie Y, Bai O, Zhang H, et al. Membrane-bound HSP70engineered myeloma cell-derived exosomes stimulate more efficient CD8 + CTL- and NK-mediated antitumour immunity than exosomes released from heat-shocked tumour cells expressing cytoplasmic HSP70. J Cell Mol Med 2010;14:2655-66.

35. Guo D, Chen Y, Wang S, et al. Exosomes from heatstressed tumour cells inhibit tumour growth by converting regulatory T cells to Th17 cells via IL-6. Immunology 2018;154:132-43.

36. Chow A, Zhou W, Liu L, et al. Macrophage immunomodulation by breast cancer-derived exosomes requires Toll-like receptor 2-mediated activation of NF- B. Sci Rep 2014;4:5750.

37. Taylor DD, Gerçel-Taylor C, Lyons KS, et al. T-cell apoptosis and suppression of T-cell receptor/CD3-zeta by Fas ligand-containing membrane vesicles shed from ovarian tumors. Clin Cancer Res 2003;9:5113-9.

38. Clayton A, Mitchell JP, Court J, et al. Human tumorderived exosomes selectively impair lymphocyte responses to interleukin-2. Cancer Res 2007;67:7458-66.

39. Wieckowski EU, Visus C, Szajnik M, et al. Tumor-derived microvesicles promote regulatory $\mathrm{T}$ cell expansion and induce apoptosis in tumor-reactive activated CD8 $+\mathrm{T}$ lymphocytes. J Immunol 2009;183:3720-30.

40. Chen G, Huang AC, Zhang W, et al. Exosomal PD-L1 contributes to immunosuppression and is associated with anti-PD-1 response. Nature 2018;560:382-6. 
41. Ning $Y$, Shen $K, W u$ Q, et al. Tumor exosomes block dendritic cells maturation to decrease the $\mathrm{T}$ cell immune response. Immunol Lett 2018;199:36-43.

42. Ludwig S, Floros T, Theodoraki MN, et al. Suppression of lymphocyte functions by plasma exosomes correlates with disease activity in patients with head and neck cancer. Clin Cancer Res 2017;23:4843-54.

43. Maybruck BT, Pfannenstiel LW, Diaz-Montero M, et al. Tumor-derived exosomes induce CD8+ T cell suppressors. J Immunother Cancer 2017;5:65.

44. Ye L, Zhang Q, Cheng Y, et al. Tumor-derived exosomal HMGB1 fosters hepatocellular carcinoma immune evasion by promoting TIM-1+ regulatory B cell expansion. J Immunother Cancer 2018;6:145.

45. Clayton A, Mitchell JP, Court J, et al. Human tumorderived exosomes down-modulate NKG2D expression. J Immunol 2008;180:7249-58.

46. Yu S, Liu C, Su K, et al. Tumor exosomes inhibit differentiation of bone marrow dendritic cells. J Immunol 2007;178:6867-75.

47. Valenti R, Huber V, Filipazzi P, et al. Human tumorreleased microvesicles promote the differentiation of myeloid cells with transforming growth factor-betamediated suppressive activity on $\mathrm{T}$ lymphocytes. Cancer Res 2006;66:9290-8.

48. Chalmin F, Ladoire S, Mignot G, et al. Membraneassociated Hsp72 from tumor-derived exosomes mediates STAT3-dependent immunosuppressive function of mouse and human myeloid-derived suppressor cells. J Clin Invest 2010;120:457-71.

49. Diao J, Yang X, Song X, et al. Exosomal Hsp70 mediates immunosuppressive activity of the myeloid-derived suppressor cells via phosphorylation of Stat3. Med Oncol 2015;32:453.

50. Wang J, Veirman K De, Beule N De, et al. The bone marrow microenvironment enhances multiple myeloma progression by exosome-mediated activation of myeloidderived suppressor cells. Oncotarget 2015;6:43992-4004.

51. Xiang X, Poliakov A, Liu C, et al. Induction of myeloidderived suppressor cells by tumor exosomes. Int J Cancer 2009; 124:2621-33.

52. Chen X, Ying X, Wang X, et al. Exosomes derived from hypoxic epithelial ovarian cancer deliver microRNA-940 to induce macrophage M2 polarization. Oncol Rep 2017;38:522-8.

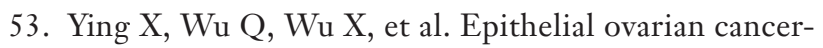
secreted exosomal miR-222-3p induces polarization of tumor-associated macrophages. Oncotarget
2016;7:43076-87.

54. Zhou J, Yang Y, Wang WW, et al. Melanoma-released exosomes directly activate the mitochondrial apoptotic pathway of CD4 + T cells through their microRNA cargo. Exp Cell Res 2018;371:364-71.

55. Zhou M, Chen J, Zhou L, et al. Pancreatic cancer derived exosomes regulate the expression of TLR4 in dendritic cells via miR-203. Cell Immunol 2014;292:65-9.

56. Ding G, Zhou L, Qian Y, et al. Pancreatic cancerderived exosomes transfer miRNAs to dendritic cells and inhibit RFXAP expression via miR-212-3p. Oncotarget 2015;6:29877-88.

57. Greening DW, Gopal SK, Xu R, et al. Exosomes and their roles in immune regulation and cancer. Semin Cell Dev Biol 2015;40:72-81.

58. Raposo G, Nijman HW, Stoorvogel W, et al. B lymphocytes secrete antigen-presenting vesicles. J Exp Med 1996;183:1161-72.

59. Admyre C, Johansson SM, Paulie S, et al. Direct exosome stimulation of peripheral human $\mathrm{T}$ cells detected by ELISPOT. Eur J Immunol 2006;36:1772-81.

60. Wolfers J, Lozier A, Raposo G, et al. Tumor-derived exosomes are a source of shared tumor rejection antigens for CTL cross-priming. Nat Med 2001;7:297-303.

61. Vega VL, Rodríguez-Silva M, Frey T, et al. Hsp70 translocates into the plasma membrane after stress and is released into the extracellular environment in a membraneassociated form that activates macrophages. J Immunol 2008;180:4299-307.

62. Cheng L, Wang Y, Huang L. Exosomes from M1polarized macrophages potentiate the cancer vaccine by creating a pro-inflammatory microenvironment in the lymph node. Mol Ther 2017;25:1665-75.

63. Poggio M, Hu T, Pai CC, et al. Suppression of exosomal PD-L1 induces systemic anti-tumor immunity and memory. Cell 2019;177:414-27.e13.

64. Cai Z, Yang F, Yu L, et al. Activated T cell exosomes promote tumor invasion via Fas signaling pathway. J Immunol 2012;188:5954-61.

65. Kim SH, Bianco N, Menon R, et al. Exosomes derived from genetically modified DC expressing FasL are anti-inflammatory and immunosuppressive. Mol Ther 2006;13:289-300.

66. Klinker MW, Lizzio V, Reed TJ, et al. Human B cellderived lymphoblastoid cell lines constitutively produce Fas ligand and secrete MHCII(+)FasL(+) killer exosomes. Front Immunol 2014;5:144.

67. Czystowska M, Han J, Szczepanski MJ, et al. IRX-2, 
a novel immunotherapeutic, protects human $\mathrm{T}$ cells from tumor-induced cell death. Cell Death Differ 2009; 16:708-18.

68. Sharma P, Mesci P, Carromeu C, et al. Exosomes regulate neurogenesis and circuit assembly. Proc Natl Acad Sci 2019;116:16086-94.

69. Zou W, Wolchok JD, Chen L. PD-L1 (B7-H1) and PD-1 pathway blockade for cancer therapy: mechanisms, response biomarkers, and combinations. Sci Transl Med 2016;8:328rv4.

70. Ashiru O, Boutet P, Fernández-Messina L, et al. Natural killer cell cytotoxicity is suppressed by exposure to the human NKG2D ligand MICA*008 that is shed by tumor cells in exosomes. Cancer Res 2010;70:481-9.

71. Fan Y, Herr F, Vernochet A, et al. Human fetal liver mesenchymal stem cell-derived exosomes impair natural killer cell function. Stem Cells Dev 2019;28:44-55.

72. Bloomston M, Frankel WL, Petrocca F, et al. MicroRNA expression patterns to differentiate pancreatic adenocarcinoma from normal pancreas and chronic pancreatitis. JAMA 2007;297:1901-8.

73. Bronte V, Brandau S, Chen SH, et al. Recommendations for myeloid-derived suppressor cell nomenclature and characterization standards. Nat Commun 2016;7:12150.

74. Gabrilovich DI, Nagaraj S. Myeloid-derived suppressor cells as regulators of the immune system. Nat Rev Immunol 2009;9:162-74.

75. Liu C, Yu S, Kappes J, et al. Expansion of spleen myeloid suppressor cells represses NK cell cytotoxicity in tumorbearing host. Blood 2007;109:4336-42.

76. Ohl K, Tenbrock K. Reactive oxygen species as regulators of MDSC-mediated immune suppression. Front Immunol 2018;9:2499.

77. Schouppe E, Van Overmeire E, Laoui D, et al. Modulation of CD8+ T-cell activation events by monocytic and granulocytic myeloid-derived suppressor cells. Immunobiology 2013;218:1385-91.

78. Taylor DD, Gercel-Taylor C. Exosomes/microvesicles: mediators of cancer-associated immunosuppressive microenvironments. Semin Immunopathol 2011;33:441-54.

79. Tian X, Shen H, Li Z, et al. Tumor-derived exosomes, myeloid-derived suppressor cells, and tumor microenvironment. J Hematol Oncol 2019;12:84.

80. Menay F, Herschlik L, De Toro J, et al. Exosomes isolated from ascites of T-cell lymphoma-bearing mice expressing surface CD24 and HSP-90 induce a tumor-specific immune response. Front Immunol 2017;8:286.

81. Malik ZA, Kott KS, Poe AJ, et al. Cardiac myocyte exosomes: stability, HSP60, and proteomics. Am J Physiol Heart Circ Physiol 2013;304:H954-65.

82. Mathew A, Bell A, Johnstone RM. Hsp-70 is closely associated with the transferrin receptor in exosomes from maturing reticulocytes. Biochem J 1995;308:823-30.

83. Corbet C, Feron O. Tumour acidosis: from the passenger to the driver's seat. Nat Rev Cancer 2017;17:577-93.

84. Brand A, Singer K, Koehl GE, et al. LDHA-associated lactic acid production blunts tumor immunosurveillance by $\mathrm{T}$ and NK cells. Cell Metab 2016;24:657-71.

85. Bellone M, Calcinotto A, Filipazzi P, et al. The acidity of the tumor microenvironment is a mechanism of immune escape that can be overcome by proton pump inhibitors. Oncoimmunology 2013;2:e22058.

86. Gupta P, Singh A, Gowda P, et al. Lactate induced HIF$1 \alpha$-PRMT1 cross talk affects MHC I expression in monocytes. Exp Cell Res 2016;347:293-300.

87. Colegio OR, Chu NQ, Szabo AL, et al. Functional polarization of tumour-associated macrophages by tumourderived lactic acid. Nature 2014;513:559-63.

88. Vermeulen M, Giordano M, Trevani AS, et al. Acidosis improves uptake of antigens and MHC class I-restricted presentation by dendritic cells. J Immunol 2004;172:3196-204.

89. Tong J, Wu WN, Kong X, et al. Acid-sensing ion channels contribute to the effect of acidosis on the function of dendritic cells. J Immunol 2011;186:3686-92.

90. Chirasani SR, Leukel P, Gottfried E, et al. Diclofenac inhibits lactate formation and efficiently counteracts local immune suppression in a murine glioma model. Int J Cancer 2013;132:843-53.

91. Nasi A, Fekete T, Krishnamurthy A, et al. Dendritic cell reprogramming by endogenously produced lactic acid. J Immunol 2013;191:3090-9.

92. Franklin RA, Liao W, Sarkar A, et al. The cellular and molecular origin of tumor-associated macrophages. Science 2014;344:921-5.

93. Peinado H, Alečković M, Lavotshkin S, et al. Melanoma exosomes educate bone marrow progenitor cells toward a pro-metastatic phenotype through MET. Nat Med 2012;18:883-91.

94. Ban JJ, Lee M, Im W, et al. Low $\mathrm{pH}$ increases the yield of exosome isolation. Biochem Biophys Res Commun 2015;461:76-9.

95. Logozzi M, Mizzoni D, Angelini DF, et al. Microenvironmental $\mathrm{pH}$ and exosome levels interplay in human cancer cell lines of different histotypes. Cancers (Basel) 2018. doi: 10.3390/cancers10100370. 
96. Jolly LA, Massoll N, Franco AT. Immune suppression mediated by myeloid and lymphoid derived immune cells in the tumor microenvironment facilitates progression of thyroid cancers driven by HrasG12V and Pten loss. J Clin Cell Immunol 2016. doi: 10.4172/2155-9899.1000451.

97. Stanton SE, Gad E, Corulli LR, et al. Tumor-associated antigens identified early in mouse mammary tumor development can be effective vaccine targets. Vaccine 2019;37:3552-61.

98. Taha EA, Ono K, Eguchi T. Roles of extracellular HSPs as biomarkers in immune surveillance and immune evasion. Int J Mol Sci 2019. doi: 10.3390/ijms20184588.

99. Proia DA, Kaufmann GF. Targeting heat-shock protein 90 (HSP90) as a complementary strategy to immune checkpoint blockade for cancer therapy. Cancer Immunol Res 2015;3:583-9.

100.Jarosz D. Hsp90: A global regulator of the genotypeto-phenotype map in cancers. Adv Cancer Res 2016;129:225-47.

Cite this article as: Boussadia Z, Zanetti C, Parolini I. Role of microenvironmental acidity and tumor exosomes in cancer immunomodulation. Transl Cancer Res 2020;9(9):5775-5786. doi: 10.21037/tcr.2020.03.69
101.Janssen N, Speigl L, Pawelec G, et al. Inhibiting HSP90 prevents the induction of myeloid-derived suppressor cells by melanoma cells. Cell Immunol 2018;327:68-76.

102. Klemm F, Joyce JA. Microenvironmental regulation of therapeutic response in cancer. Trends in Cell Biology 2015;25:198-213.

103. Marchiq I, Pouysségur J. Hypoxia, cancer metabolism and the therapeutic benefit of targeting lactate/ $\mathrm{H}(+)$ symporters. J Mol Med (Berl) 2016;94:155-71.

104. De Henau O, Rausch M, Winkler D, et al. Overcoming resistance to checkpoint blockade therapy by targeting PI3K $\gamma$ in myeloid cells. Nature 2016;539:443-7.

105.Robbins PD, Morelli AE. Regulation of immune responses by extracellular vesicles. Nat Rev Immunol 2014;14:195-208.

106. Barros FM, Carneiro F, Machado JC, et al. Exosomes and immune response in cancer: friends or foes? Front Immunol 2018;9:730. 